\title{
Full transmission through perfect-conductor subwavelength hole arrays
}

\author{
F. J. García de Abajo, ${ }^{1,2}$ R. Gómez-Medina, ${ }^{2,3}$ and J. J. Sáenz ${ }^{3}$ \\ ${ }^{1}$ Unidad de Física de Materiales CSIC-UPV/EHU Aptdo. 1072, 20080 San Sebastian, Spain \\ ${ }^{2}$ Donostia International Physics Center (DIPC), Paseo Manuel de Lardizábal, 4, 20018 San Sebastian, Spain \\ ${ }^{3}$ Departamento de Física de la Materia Condensada, Universidad Autónoma de Madrid, Cantoblanco, 28049 Madrid, Spain
}

(Received 8 November 2004; published 12 July 2005)

\begin{abstract}
Light transmission through 2D subwavelength hole arrays in perfect-conductor films is shown to be complete $(100 \%)$ at some resonant wavelengths even for arbitrarily narrow holes. Conversely, the reflection on a 2D planar array of nonabsorbing scatterers is shown to be complete at some wavelengths regardless how small the scatterers are. These results are proven analytically and corroborated by rigorous numerical solution of Maxwell's equations. This work supports the central role played by dynamical diffraction during light transmission through subwavelength hole arrays and it provides a systematics to analyze more complex geometries and many of the features observed in connection with transmission through hole arrays.
\end{abstract}

DOI: 10.1103/PhysRevE.72.016608

The fraction of light transmitted through a periodic array of subwavelength holes perforated in a metallic film has been shown to exceed the open fraction occupied by the holes at certain wavelengths related to the array periodicity [1], and the observed transmission can be anomalously large as compared to well established predictions for isolated holes [2]. This phenomenon has triggered an intense amount of activity on both experimental [1,3-9] and theoretical [4,5,10-15] fronts. While the first observations were made in the near infrared (NIR) [1,4-7], the effect has been recently corroborated at $\mathrm{THz}$ frequencies $[3,8,9]$, where claims have been made that the transmission is even larger $[8,9]$. Two different kinds of complementary interpretations of the enhanced transmission have been proposed so far, depending on whether surface plasmons [1,7,11-14] or dynamical diffraction resonances $[5,10,15]$ are invoked as the origin of the effect.

In this paper, Babinet's principle [16] is used to study light transmission through hole arrays in perfect-conductor thin films (PCTFs) by relating it to the reflection on planar arrays of metallic disks, which are solved from the multipolar polarizability of single disks. It is found that transmission reaches $100 \%$ at some resonant wavelengths regardless how small the holes are, while the complementary system exhibits perfect reflection resonances for arbitrarily small disks. These resonances are associated to divergences in the coherent interaction among disks or holes, as in the Wood anomalies [17]. A quasi-bound-state (QBS) close to the onset of the first propagating diffraction channel (i.e., close to a Rayleigh frequency) shows up due to the interaction of the scattered field with diffraction modes. The coupling between the incoming field and the QBS leads to transmission or reflection resonances which display characteristic Fano-line shapes [18]. This is rigorously proven by analytically solving the small-hole limit and also via full numerical solution of Maxwell's equations. A typical result of our analysis is advanced in Fig. 1, showing full transmission at wavelengths $\lambda$ immediately above the lattice period $a$. The theory of hole arrays in PCTFs traces back to the works of Eggimann, Collin, and Chen [19], and McPhedran et al. [20]. The latter presents comprehensive comparisons with experiments, demonstrating the existence of $100 \%$ transmission peaks at wavelengths
PACS number(s): 42.25.Fx, 41.20.Jb, 42.79.Ag, 78.66.Bz

immediately above the period. Here, we extend the result of full transmission to arbitrarily small holes.

Babinet's principle [16] provides a powerful tool for analyzing complex perfect-conductor planar structures. For instance, it implies that any self-complementary 2D-patterned flat screen (e.g., a chessboard of square apertures) reflects and transmits exactly half of the unpolarized light coming at normal incidence for any wavelength. Also, a simple derivation of Bethe's result for a small isolated hole in a metallic screen [2] follows from this principle: for incidence normal to a metallic disk of diameter $D$, the electric polarizability reduces to $\alpha_{E}=D^{3} / 6 \pi$, while the magnetic response vanishes altogether [16], leading to a forward scattering cross section given by $\sigma /\left(\pi D^{2} / 4\right)=4(k D)^{4} / 27 \pi^{2}$ (normalized to the disk area), as calculated from the Poynting vector in the forward hemisphere, where $k=2 \pi / \lambda$; now, Babinet's principle implies that this result must coincide with the transmission cross section of a hole of the same diameter, in perfect agreement with Bethe's formula [2].

For hole arrays in PCTFs, Babinet's principle connects their transmittance to the reflectance of the complementary geometries consisting of planar arrays of perfect-conductor thin disks [19]. In the small-hole limit, this can be obtained by self-consistently solving for the electric dipoles induced on the disks, $\mathbf{p}_{\mathbf{R}}=\alpha_{E} \mathbf{E}^{\text {inc }}(\mathbf{R})+\alpha_{E} \sum_{\mathbf{R}^{\prime} \neq \mathbf{R}} G_{E}^{\mathbf{R} \mathbf{R}^{\prime}} \mathbf{p}_{\mathbf{R}^{\prime}}$, where $\mathbf{R}$ and $\mathbf{R}^{\prime}$ label disk sites, $\alpha_{E}$ is the electric polarizability of the disks, and $G_{E}^{\mathbf{R R}^{\prime}}$ is the dipole-dipole interaction dyadic. At normal incidence on periodic arrays, the polarization is the same in all disks, parallel to the incident electric field $\mathbf{E}^{\text {inc }}(\| \hat{\mathbf{x}})$, and given by

$$
p=\frac{1}{\frac{1}{\alpha_{E}}-G_{E}},
$$

where

$$
G_{E}=\sum_{\mathbf{R} \neq 0}\left(k^{2}+\partial_{x x}^{2}\right) e^{i k R} / R .
$$

Below the threshold of the first diffraction channel, the farfield induced by the lattice of identical dipoles is readily 

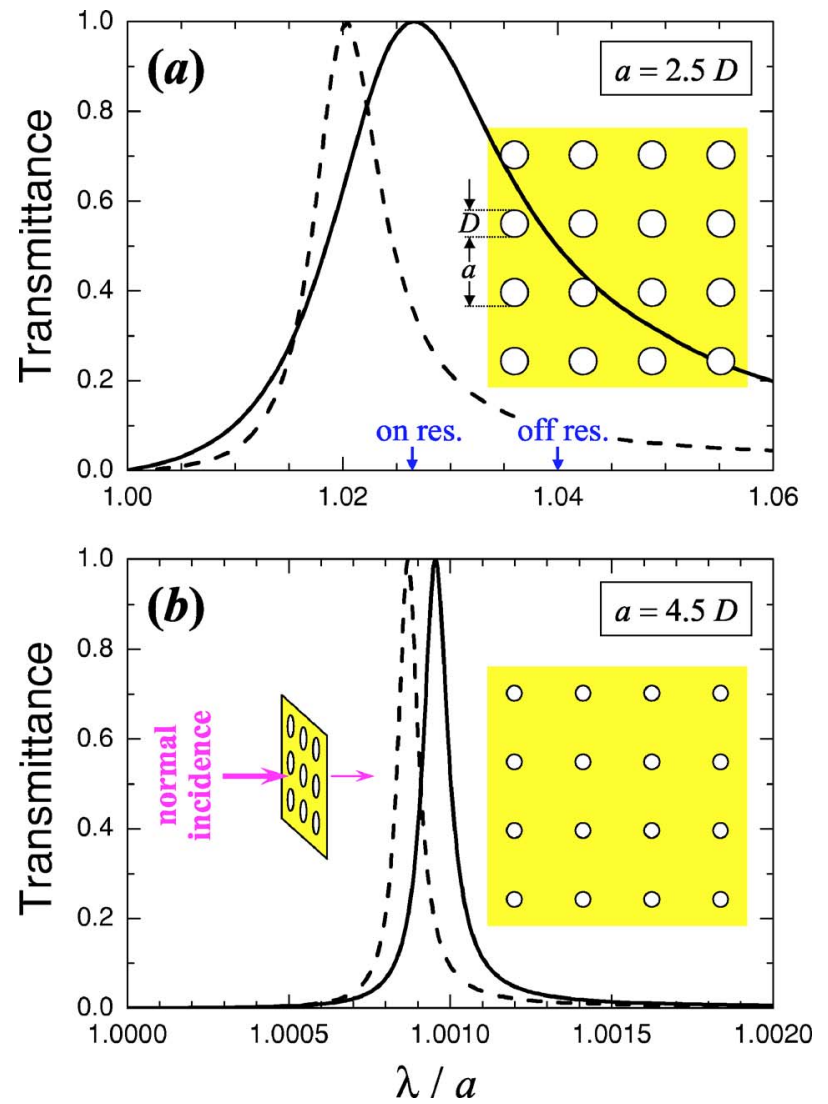

FIG. 1. (Color online) Light transmittance through a square array of circular holes perforated in a thin perfect-conductor screen as a function of wavelength $\lambda$ normalized to the lattice constant $a$ for two different diameters of the holes: (a) $D=a / 2.5$ and (b) $D$ $=a / 4.5$. The light is impinging normal to the screen and a $100 \%$ transmission peak is obtained in both cases. Solid curves: full numerical results. Broken curves: analytical model of Eq. (3).

calculated as $E^{\mathrm{ind}}=(2 \pi \mathrm{i} k / A) p e^{i k|z|}$, where $A$ is the area of the unit cell and $z$ is chosen perpendicular to the lattice plane. From here, one obtains the transmittance $\mid 1$ $+\left.(2 \pi i k / A) p\right|^{2}$ and reflectance $|(2 \pi k / A) p|^{2}$ of the disk array, which have to sum 1 because perfect conductors cannot dissipate energy. This condition leads to $\operatorname{Im}\left\{1 / \alpha_{E}-G_{E}\right\}=$ $-2 \pi k / A$, which generalizes the optical theorem for nonabsorbing particles, $\operatorname{Im}\left\{-1 / \alpha_{E}\right\}=2 k^{3} / 3$. We can then write the reflectance of the disk array, which equals the transmittance of the complementary hole array from Babinet's principle, as

$$
T=\frac{1}{1+\left(\frac{A}{2 \pi k} \operatorname{Re}\left\{\alpha_{E}^{-1}-G_{E}\right\}\right)^{2}} .
$$

Like in the Wood anomalies [15,17], the structure factor $G_{E}$ diverges when one of the reflected beams goes grazing, and in particular near $\lambda=a$, the period for square lattices. More precisely, $\operatorname{Re}\left\{G_{E} a^{3}\right\}=4 \pi^{2} \sqrt{2} / \sqrt{\lambda / a-1}+C(\lambda / a)$, with $\lambda>a$, where the first term is derived analytically from the divergent terms of the sum over reciprocal lattice vectors into which Eq. (2) can be recast, and $C(\theta) \approx 35 \exp [-22(\theta-1)]-118$ is a smooth fit to the remaining nondivergent terms.

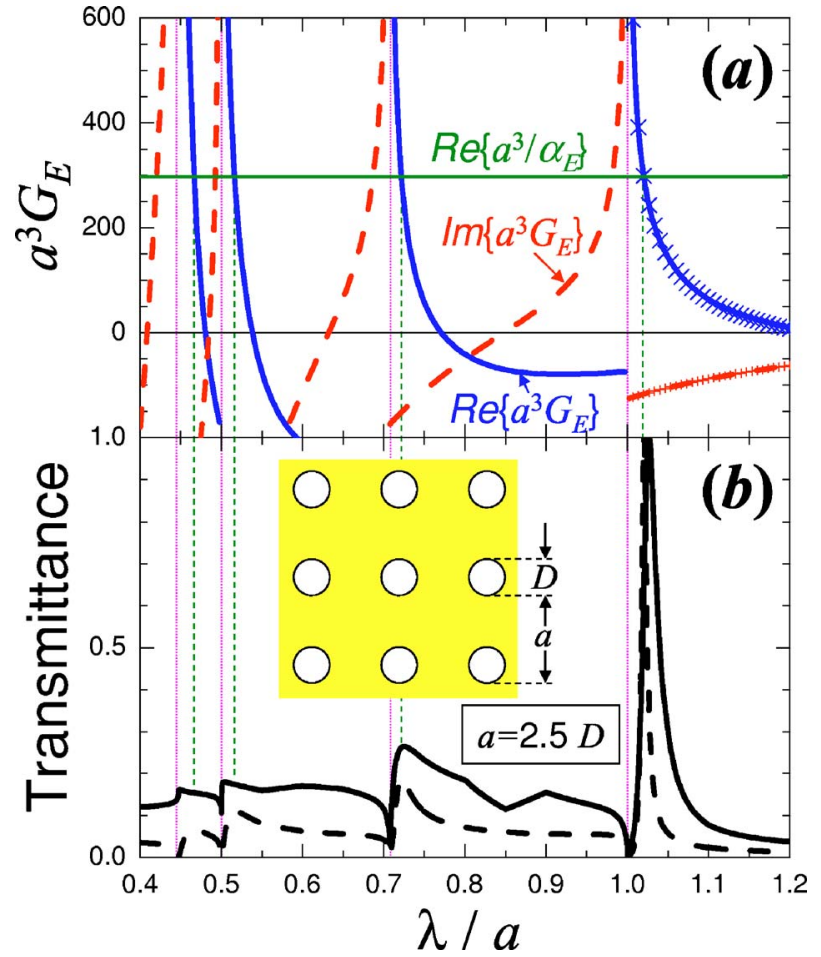

FIG. 2. (Color online) (a) Wavelength dependence of $G_{E}$ [Eq. (2)] and determination of transmission maxima and minima: the solid (broken) curve represents the real (imaginary) part of $G_{E}$ for a square lattice under normal incidence. Transmission minima (see vertical dotted lines) result from divergences of $G_{E}$, while transmission maxima (vertical dashed lines) are derived from the condition that $\operatorname{Re}\left\{G_{E}\right\}$ equals the inverse of the polarizability of the hole. (b) Transmittance under the same conditions as in Fig. 1(a) over a wider wavelength range.

Obviously, $G_{E}$ diverges as $\lambda \rightarrow a^{+}$, and thus, given an arbitrarily-small hole diameter, there is always one wavelength $\lambda>a$ for which the second term in the denominator of Eq. (3) vanishes, implying that the transmittance (reflectance) becomes $100 \%$ at that frequency for the array of holes (disks), as shown in Fig. 1 (dashed curves). This is graphically illustrated in Fig. 2, where $\operatorname{Re}\left\{G_{E}\right\}$ has been represented [Fig. 2(a)] and compared with $\operatorname{Re}\left\{1 / \alpha_{E}\right\}$ (horizontal line). The crossing points of these two agree very well with the transmission maxima predicted by the analytical Eq. (3) [dashed curve in Fig. 2(b)].

This divergence of $G_{E}$ is the same one that produces Wood's anomalies and is related to the geometry of the system. For disks, this is simply coming from the accumulation of in-phase contributions from long-distance coherent multiple scattering (MS) $[5,10,15]$. In the complementary structure (hole arrays), the same systematics is at work via Babinet's principle, as described above. Moreover, the reflectance (transmittance) of the disk (hole) array [Eq. (3)] exhibits the same behavior as Fano reflection resonances, in agreement with reported descriptions of the mechanism of transmission enhancement [15]. In general, Fano resonances [18] occur when the energy (frequency) of the incoming wave is tuned to the energy (frequency) of a QBS. Our QBS is confined to the lattice and partially leaking into the continuum of 


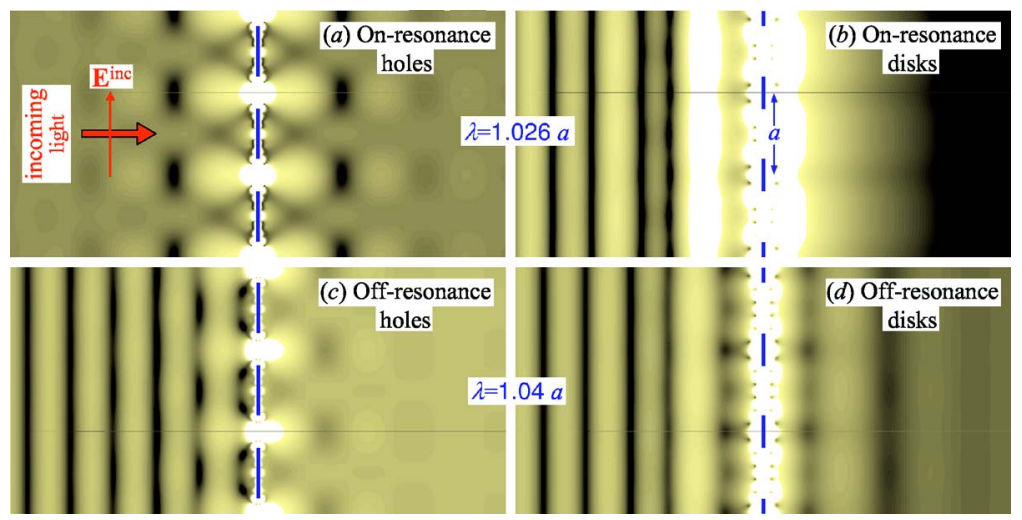

FIG. 3. (Color online) (a) and (c): squared electric field in log scale for light incident normal to the hole array considered in Fig. 1(a), under resonance and nonresonance conditions, respectively (see arrows in [Fig. 1(a)]. The plane of representation is parallel both to the screen normal and to one of the lattice vectors, and it intersects the centers of a row of holes. The metallic screen and its holes are represented by vertical broken lines. (b) and (d): same for the disk array defined as the complementary screen with respect to that of (a) and (c).

vacuum light states, giving rise to some broadening of the transmission peaks, as observed in Fig. 1.

For perfect-conductor disks or hole arrays, we can then consider the transmission enhancement as a consequence of the coupling of the incoming field with a QBS of geometrical origin. Plasmon-free perfect conductors are thus capable of exhibiting extraordinary transmission (actually 100\%) for arrays of holes however small.

We have also carried out a rigorous solution of Maxwell's equations that is represented in Fig. 1 by solid curves. In our method, we first obtain the multipolar scattering matrix of a disk of the same diameter as our holes using the boundary element method [21]. Then, we solve the problem of a lattice of disks, which is the exact complementary screen geometry of our hole array, using the layer-KKR approach [22]. Finally, the solution for the hole array is related to the solution for the disk array using Babinet's principle, and in particular, the transmittance for $s(p)$ polarization in the former equals the reflectance for $p(s)$ polarization in the latter.

Figure 1 shows results for two different ratios of the diameter $D$ to the lattice constant $a$. The analytical model of Eq. (3) explains well the shift of the transmission resonance wavelength towards $a$ and its narrowing with decreasing $D / a$. Actually, Eq. (3) works better for smaller holes, since it is based upon the dipolar part of their response, and it should be exact in the $D / a \rightarrow 0$ limit, although higher perfectness of hole shape, size, and position will be necessary to observe narrower resonances.

Of course, the derivation of Eq. (3) fails when more than one transmitted beam is present: $100 \%$ transmission can only occur for $\lambda>a$ in our square lattices. This is shown in the transmission maxima of Fig. 2(b) for $\lambda<a$, where nonnormal beams take part of the light flux, and where Eq. (3) (broken curve) is shown to reproduce qualitatively our rigorous calculations (solid curve).

A closer look into the near field is shown in Fig. 3 for on-resonance [(a) and (b)] and off-resonance [(c) and (d)] scenarios in a hole array [(a) and (c)] and in its complementary disk array [(b) and (d)]. The effect of the on-resonance hole array [Fig. 3(a)] extends up to a distance of the order of the lattice period $a$, beyond which the electric field strength is quite uniform. For the on-resonance disk array [Fig. 3(b)], there is an evanescent field beyond the plane of the disks and total reflection establishes an interference pattern on the incoming-light side. The off-resonance scenario exhibits in- terference of incoming and partly-reflected light, as well as partial light transmission. It should be noticed that the field near the hole array is more intense on resonance (this is a signature of a QBS) as a result of stronger $\mathrm{MS}$, and this anticipates stronger absorption if lossy materials are employed rather than perfect conductors, in agreement with recent experiments [7].

When light is coming under oblique incidence, the above model has to be supplemented by adding a phase $\exp \left\{i \mathbf{k}_{\|}^{\text {inc }} \cdot \mathbf{R}\right\}$ to the dipoles induced on the different hole sites R. And more importantly, both magnetic and electric responses will play a role. In fact, any perfect-conductor planar object of finite extension such as our disks can sustain induced currents and charges only within the plane where it is

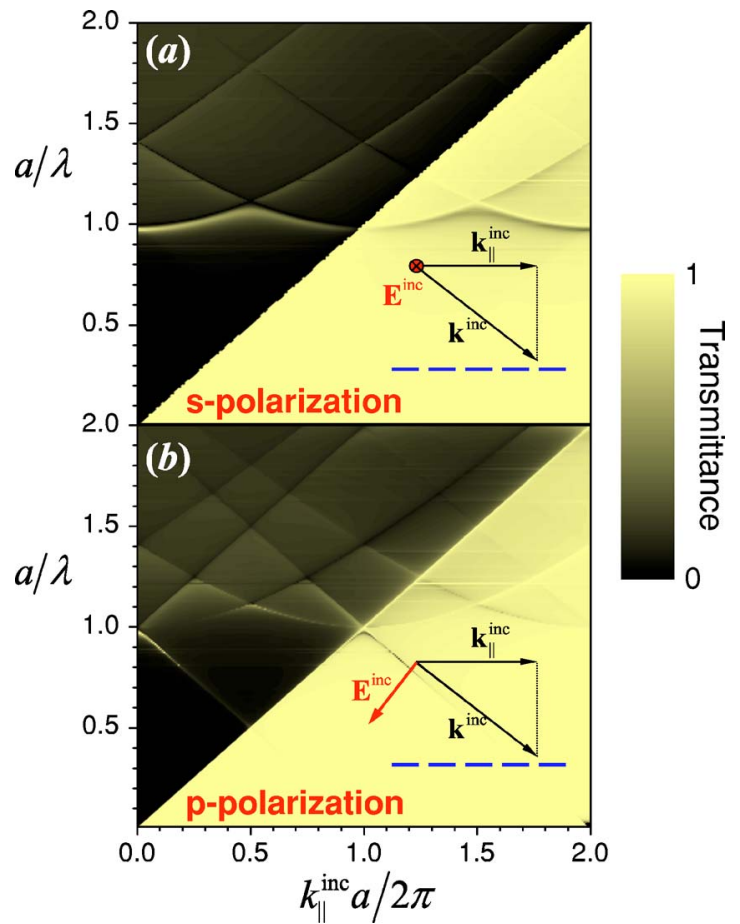

FIG. 4. (Color online) Angular dependence of the transmission of $s$ - and $p$-polarized light [region above the light line in (a) and (b), respectively] incident upon a perfect-conductor screen perforated by an array of circular holes of diameter $D=a / 2.5$ arranged in a square lattice of constant $a$. Resonances outside the light cone are also shown by plotting the transmitted far field when the screen is exposed to an incident evanescent wave. 
contained, leading to induced electric dipoles parallel to the plane and induced magnetic dipoles perpendicular to it in the long wavelength limit. The latter can only be excited under oblique incidence.

In particular, the magnetic polarizability of a disk, $\alpha_{M}=$ $-D^{3} / 12 \pi$, reacts only to normal magnetic field components. The disks in a lattice are coupled via $G_{M}$ $=\Sigma_{\mathbf{R} \neq 0} \exp \left\{i \mathbf{k}_{\|}^{\text {inc }} \cdot \mathbf{R}\right\}\left(k^{2}+\partial_{z z}^{2}\right) e^{i k R} / R$ to produce perpendicular magnetic dipoles given by the magnetic counterpart of Eq. (1). In fact, there are two uncoupled sets of resonances in disk arrays that respond (i) to a simultaneous mixture of external perpendicular magnetic fields and parallel electric fields (ii) and to external parallel electric fields alone, respectively. For $s$-polarized incident light $\left(\mathbf{E}^{\text {inc }}\right.$ parallel to the screen) only the latter can be excited, whereas $p$-polarized light can couple to both types of resonances, as illustrated in Fig. 4 via the dependence of the transmittance on incidentlight wavelength and parallel momentum. The region above the light line $\left(k>k_{\|}^{\text {inc }}\right)$ shows transmission maxima (bright areas) and minima (right above the maxima), with $100 \%$ transmission achieved only for $p$ polarization and $\lambda>a$. As expected, $p$-polarized light couples to more resonances [of types (i) and (ii)] than s-polarized light [only resonances of type (ii)]. This conclusions might be relevant to explain recent measurements of polarization- and angle-resolved transmission in thick arrays of real metal [7] with similar qualitative behavior as that of Fig. 4.

Our methods can be trivially extended to other geometries like finite arrays [8] and more complex hole shapes [4,6]. In particular, the asymmetry of the polarizability tensor of a rectangular metallic plate, which is larger along its long axis, is consistent with the stronger red shift of the transmitted maxima observed for lattices of square holes [4] as compared to circular holes of similar size. Also, new metamaterials composed of layers of hole arrays and disk arrays could be analyzed by straightforward generalization of the methods presented above.

In real metals, the dispersion relation of surface plasmons deviates from the light line and then the transmission enhancement observed in the NIR is the result of in-phase MS mediated by electromagnetic propagation via these modes.

Furthermore, the metal films are supported on glass substrates in many experiments $[1,4,7]$. This analysis can be easily extended to a PCTF surrounded by dielectrics of different index of refraction on either side as shown by Dawes et al. [23], in which case full transmission resonances occur only when the wavelength in the higher-index region is above the smallest reciprocal surface lattice vector of the array (e.g., near $\lambda \approx 1.4 a$ for a square lattice supported on glass), and the effective polarizability of the hole differs from the symmetric case considered above, but apart from this the long-wavelength analysis presented here remains the same.

In summary, we have shown that the extraordinary transmission of light through hole arrays in PCTFs can be fully explained in simple terms by invoking Babinet's principle. The existence of $100 \%$ transmission maxima has been established, regardless how small the holes are. The origin of full transmission can be found in phase accumulation during long-range dipole-dipole interaction among holes when the wavelength is close to the onset of the first diffraction order. Potential application to narrow filters can be envisioned in the microwave and $\mathrm{THz}$ regimes, since the transmission resonances are increasingly sharper as the holes become smaller.
[1] T. W. Ebbesen et al., Nature (London) 391, 667 (1998);H. F. Ghaemi et al., Phys. Rev. B 58, 6779 (1998).

[2] H. A. Bethe, Phys. Rev. 66, 163 (1944).

[3] J. Gómez-Rivas et al., Phys. Rev. B 68, 201306(R) (2003).

[4] K. J. Klein Koerkamp et al., Phys. Rev. Lett. 92, 183901 (2004).

[5] H. J. Lezec and T. Thio, Opt. Express 12, 3629 (2004).

[6] R. Gordon et al., Phys. Rev. Lett. 92, 037401 (2004).

[7] W. L. Barnes et al., Phys. Rev. Lett. 92, 107401 (2004).

[8] F. Miyamaru and M. Hangyo, Appl. Phys. Lett. 84, 2742 (2004).

[9] H. Cao and A. Nahata, Opt. Express 12, 1004 (2004).

[10] M. M. J. Treacy, Appl. Phys. Lett. 75, 606 (1999); , Phys. Rev. B 66, 195105 (2002).

[11] E. Popov et al., Phys. Rev. B 62, 16100 (2000).

[12] L. Martín-Moreno et al., Phys. Rev. Lett. 86, 1114 (2001).

[13] R. Wannemacher, Opt. Commun. 195, 107 (2001).

[14] L. Salomon et al., Phys. Rev. Lett. 86, 1110 (2001).
[15] M. Sarrazin, J.-P. Vigneron, and J.-M. Vigoureux, Phys. Rev. B 67, 085415 (2003).

[16] J. D. Jackson, Classical Electrodynamics (Wiley, New York, 1975).

[17] R. W. Wood, Phys. Rev. 48, 928 (1935).

[18] U. Fano, Phys. Rev. 124, 1866 (1961).

[19] W. H. Eggimann and R. E. Collin, IRE Trans. Microwave Theory Tech. 10, 528 (1962); C. C. Chen, IEEE Trans. Microwave Theory Tech. 19, 475 (1971).

[20] R. C. McPhedran et al., in Electromagnetic Theory of Gratings, edited by R. Petit (Springer, Berlin, 1980), p.227.

[21] F. J. García de Abajo and A. Howie, Phys. Rev. Lett. 80, 5180 (1998).

[22] N. Stefanou, V. Yannopapas, and A. Modinos, Comput. Phys. Commun. 113, 49 (1998); 132, 189 (2000).

[23] D. H. Dawes, R. C. McPhedran, and L. B. Whitbourn, Appl. Opt. 28, 3498 (1989). 